\title{
iLIF: illumination by Laser-Induced Fluorescence for single flash imaging on a nanoseconds timescale
}

\author{
Arjan van der Bos $\cdot$ Aaldert Zijlstra • \\ Erik Gelderblom • Michel Versluis
}

Received: 28 February 2011/Revised: 1 June 2011/ Accepted: 10 June 2011/Published online: 28 June 2011

(C) The Author(s) 2011. This article is published with open access at Springerlink.com

\begin{abstract}
The challenge in visualizing fast microscale fluid motion phenomena is to record high-quality images free of motion-blur. Here, we present an illumination technique based on laser-induced fluorescence which delivers high-intensity light pulses of $7 \mathrm{~ns}$. The light source consists of a Q-switched Nd:YAG laser and a laser dye solution incorporated into a total internal reflection lens, resulting in a uni-directional light beam with a millimetersized circular aperture and $3^{\circ}$ divergence. The laser coherence, considered undesirable for imaging purposes, is reduced while maintaining a nanoseconds pulse duration. The properties of the illumination by laser-induced fluorescence $(i L I F)$ are quantified, and a comparison is made with other high-intensity pulsed and continuous light sources.
\end{abstract}

\section{Introduction}

It has been a challenge throughout the last century to freeze fast fluid motion phenomena onto a single image and, in such a way, distinguish the finest details at the smallest length scales. One of the first to address this challenge was A.M. Worthington (1895) when he used a spark to visualize a splash of milk. Worthington already predicted that flash recording would become of great importance in scientific research, but it was not until the work of Edgerton and Killian (1979) that the scientific utilization of flash

A. van der Bos $(\varangle) \cdot$ A. Zijlstra · E. Gelderblom · M. Versluis Physics of Fluids group, Faculty of Science and Technology and Burgers Center of Fluid Dynamics, MESA+ Institute for Nanotechnology and Institute of Mechanics, University of Twente, P.O. Box 217, 7500 AE Enschede, The Netherlands e-mail: J.A.vanderBos@alumnus.utwente.nl photography became widely accepted. In the last decade, digital camera technology has evolved rapidly, and highspeed visualization has become a valuable experimental method, see e.g. Thoroddsen et al. (2008). At the same time, illumination techniques continued to develop, from high-intensity Xenon light sources to low-cost, highintensity LEDs, both of which can be used for pulsed as well as continuous illumination.

In microfluidics, a wide range of phenomena are studied, including the dynamics of droplets, bubbles and particles. Despite the small sizes of these objects, the associated velocities are usually in the order of meters per second. Hence, to achieve accurate visualization not only a high spatial resolution but also a high temporal resolution is required. For this purpose, one can either employ a camera with a short exposure time or a light source capable of emitting flashes of short duration. The first option generally requires a fast (and expensive) high-speed camera, which is often limited in its pixel resolution (Thoroddsen et al. 2008). Therefore, flash photography is the preferred method for experiments where high-resolution single images are required, since the method does not require a fast shutter and can in principle be performed with any type of camera. Furthermore, the pulse duration of flash illumination sources can easily be shorter than the shortest exposure time of a high-speed camera. On the other hand, the repetition rate of high-intensity flash light sources is often limited to several kilohertz, making these sources unsuitable for recording multiple consecutive frames at microseconds or shorter timescales.

Nowadays, several types of nanosecond light sources are available, which can deliver a considerable amount of light within a few nanoseconds. There are, for example, affordable solutions with high-intensity LEDs (Vasiliev et al. 2000; Scholfield and Murdock 1987; Lubsandorzhiev 
et al. 2009), which can deliver up to $10^{12}$ photons per flash of $2 \mathrm{~ns}$, thus close to $1 \mu \mathrm{J}$ per flash. A comparable, but more expensive solution, is using high-intensity spark plugs (Miyashiro and Grönig 1985; High Speed Photo Systeme). However, when it comes to emitting ultrashort high-intensity light flashes, a pulsed laser is clearly the most effective. The duration of laser pulses are typically in the order of nanoseconds, but can be as short as attoseconds (Takahashi et al. 2010), while the energy per pulse is in the millijoule range or higher. Lasers can be used directly for flash illumination, as was done for example in Dong et al. (2006) and Juhasz et al. (1996), but there are some major drawbacks associated with a fully coherent light source. The light emitted by a laser has a very narrow bandwidth and has a long coherence length, resulting in diffraction fringes and laser speckle patterns in the recorded images (Juhasz et al. 1996). Additionally, spurious reflection of the laser beam has the potential risk of causing permanent damage to the camera sensor. Methods to suppress these effects are predominantly based on reducing the spatial coherence the light, for example by applying rotating or vibrating optical elements like fibers, wobblers and diffusers (Iwai and Asakura 1996). A good example to obtain this was proposed by Dingel and Kawata (1993), where the laser light is coupled into multiple fibers with different lengths, after which the light from the separate fibers is recombined and passes through a spatial diffuser (Nanophoton). For a laser pulse, however, a more effective method is to decrease its temporal coherence. One interesting approach to achieve this is through excitation of a fluorescent laser dye. Here, the pulse duration is practically unaltered, but the energy of the pulse is redistributed into a broader optical bandwidth at higher wavelengths. This technique has proven to be very effective and was already applied in shadowgraph experiments (Zijlstra and Ohl 2008) and is closely related to fluorescent flow visualization described in Rau et al. (2006) and Shan et al. (2004). A disadvantage is that the cuvette containing the dye can act as a secondary laser cavity (Schäfer et al. 1966), thereby partially restoring the coherent nature of the pulse (see Fig. 1). Additionally, relatively high laser energies were required to obtain acceptable illumination intensities, as a result of high losses due to the omni-directionality of the fluorescence emission.

Here, we address these issues using a modified single flash illumination technique based on laser-induced fluorescence. The obtained illumination is ideally suited for flash photography in experiments involving timescales of order microseconds in conjunction with length scales of order micrometer. To validate the technique, its performance is compared in a standard imaging setup with a range of high-end commercially available light sources, both pulsed and continuous.

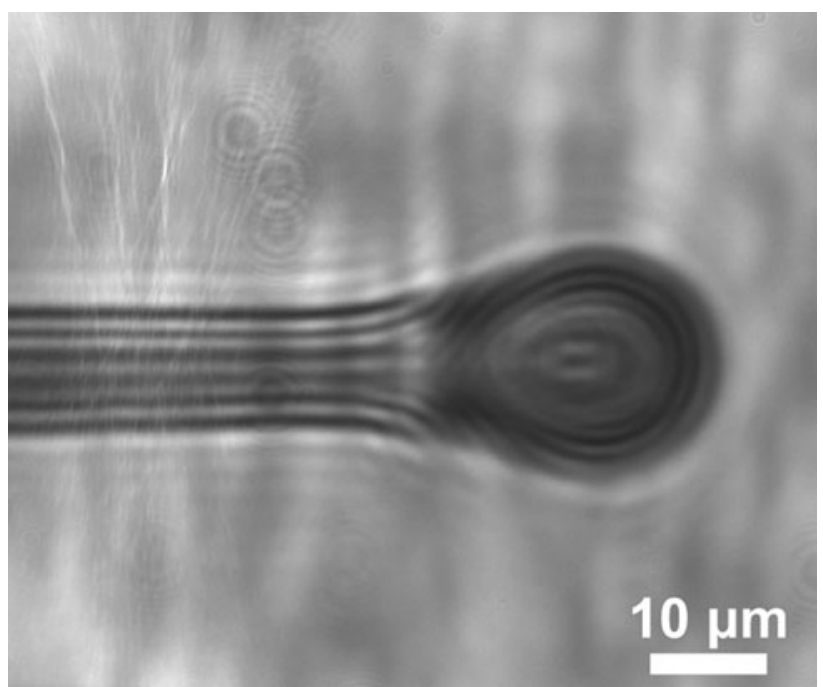

Fig. 1 The head of an inkjet droplet moving from left to right with a velocity close to $6 \mathrm{~m} / \mathrm{s}$. This image was made using the illumination where the laser dye is contained in a cuvette as described in Zijlstra and Ohl (2008). The image shows a speckle pattern in the background and interference patterns of the light passing through and around the droplet

\section{Criteria for high-speed flash photography}

The application of flash photography in experiments is primarily aimed at obtaining precise information about the position and dimensions of the studied object at a certain instant in time. It is therefore of prime importance to capture the smallest details in both temporal- and spatial resolution at high contrast. To define this more quantitatively, three criteria are stated. These are the spatial Nyquist criterion, maximizing the signal-to-noise ratio of the $\mathrm{CCD}$ and minimizing motion-blur.

First, assuming that a microscope objective with the highest possible numerical aperture is chosen for the experiment, the effective optical magnification $M_{\mathrm{eff}}$ must be sufficient to avoid undersampling of the image with respect to the spatial resolution $r$ as defined by the Rayleigh-criterion (Davidson and Abramowitz 2002). The spatial Nyquist criterion requires that at least 2 pixels lay within $r M_{\text {eff }}$ hence it follows that the magnification must be such that

$M_{\text {eff }}>\frac{2 d}{r}$

where $d$ is the pixel size. Larger magnifications allow denser spatial sampling (known as oversampling) but also result in a smaller field-of-view and lower image brightness. The magnification is therefore limited by the optimum size of the object-image and the available illumination intensity. 
Secondly, to ensure maximum image contrast, the intensity of the illumination should be adjusted to cover the full dynamic range of the camera sensor. The extent to which the intensity can be varied is, however, limited due to the reciprocal relationship between illumination intensity and exposure time. To clarify, an image with a certain fixed brightness can be obtained with, either low light conditions and longer exposure times, or vice versa. In the situation where an object is motionless both settings result in identical images. However if the object of interest is moving, the image becomes susceptible to motion-blur. This undesired effect causes a smeared appearance of the image of the object due to its displacement during the time the image is recorded.

Minimizing motion-blur constitutes the third criterion that is required to accurately capture a single high-resolution image of a moving object and this can be achieved by adjusting the temporal resolution of the imaging system. As discussed, this temporal resolution is determined either by the duration of the illumination pulse (indicated by $\tau_{p}$ ) or by the camera exposure time $\tau_{c}$, which is the time duration during which the mechanical or electronic shutter of the camera is opened to expose the sensor to an illuminated object. Evidently, the actual temporal resolution $\tau$ will be the shortest of both durations, hence

$\tau=\min \left(\tau_{p}, \tau_{c}\right)$.

If we now define $\epsilon$ as being the displacement in pixels of the object moving with velocity $U$ during time $\tau$ :

$\epsilon=\tau \frac{M_{\mathrm{eff}} U}{d}$

motion-blur will be minimal if $\epsilon \leq 1$. In most experiments, the object velocity, magnification and pixel size are predetermined and motion-blur is minimized by choosing an illumination or exposure time such that

$\tau \leq \frac{d}{M_{\mathrm{eff}} U}$.

\section{Illumination by laser-induced fluorescence}

To obtain maximum fluorescence efficiency while minimizing coherence, we constructed a laser dye lens. The core of a total internal reflection lens (TIR-lens) where normally a high-power LED can be placed was filled with a laser dye (Fig. 2). This dye consisted of a fully saturated solution of LDS 698 (Exciton Inc., Dayton, U.S.) in ethanol. The TIR-lens (L2-Optics) is an acrylic collimator with a total beam divergence of $3^{\circ}$. The dye was excited by a Nd:YAG laser (Solo PIV, New Wave) emitting a $6.5 \mathrm{~ns}$ pulse (full width at half maximum, see Fig. 3) with a maximum energy of $100 \mathrm{~mJ}$ at $532 \mathrm{~nm}$. This configuration

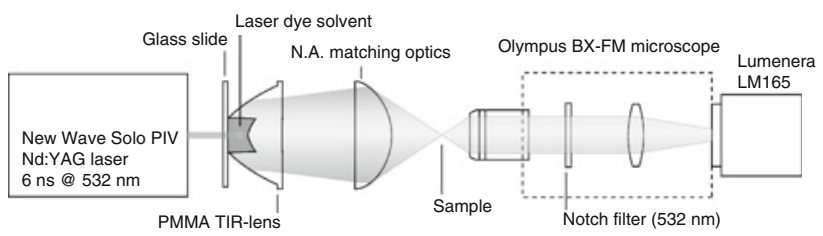

Fig. 2 Typical application of illumination with laser dye fluorescence. In the illumination setup, a laser excites the dye after which the fluorescent light from the cavity is collected and focused through a collimation lens onto a microscope. To filter out any residual laser light, a notch filter (Semrock NF01-532U-25) is used

allows the greater part of the laser pulse to be absorbed, reemitted and subsequently reflected in the forward direction thus promoting the reduction in the temporal coherence of the pulse.

To confirm the applicability of this setup for illumination by laser-induced fluorescence (iLIF), the pulse length and spectrum were measured. To measure the pulse length, a photodiode (Thorlabs-DET 210) was mounted directly after the TIR-lens and connected to an oscilloscope (Tektronix TDS5034B), resulting in a typical rise time of the measuring system of $1.2 \mathrm{~ns}$. The laser and oscilloscope were triggered with a pulse/delay generator (Berkeley Nucleonics model 565) with an accuracy of 250 ps. The measurement was carried out with and without a notch filter in between the lens and photodiode. As shown in Fig. 3 the $i L I F$ does not result in a noticeable difference in pulse length with respect to the laser. The influence of the notch filter on the $i L I F$-pulse shape was also found to be negligible.

The spectral characteristics of the $i L I F$ pulse were determined using a high-resolution fiber optic spectrometer (AVASPEC 3648, FWHM resolution: $0.32 \mathrm{~nm}$ ). Fig. 4 shows the broad spectrum of the fluorescence light. The spectrum was recorded with the notch filter in front of the spectrometer. However, measurements without the notch filter also did not show a peak at $532 \mathrm{~nm}$, indicating that most of the laser energy is absorbed by the laser dye.

\section{Comparison with other light sources}

To quantify the performance of the laser-induced fluorescence illumination, a comparison was made with several high-intensity light sources, typically used for high-speed imaging of microscopic events. An overview of the light sources, both pulsed and continuous, is given in Table 1 . All continuous light sources have an optical fiber, glass or liquid core with a diameter of $4-8 \mathrm{~mm}$, to deliver the light to the sample, except for the LED's and the continuous xenon light source. The LED's are mounted in a TIR-lens with a divergence of $3^{\circ}$, comparable with the TIR-lens used 


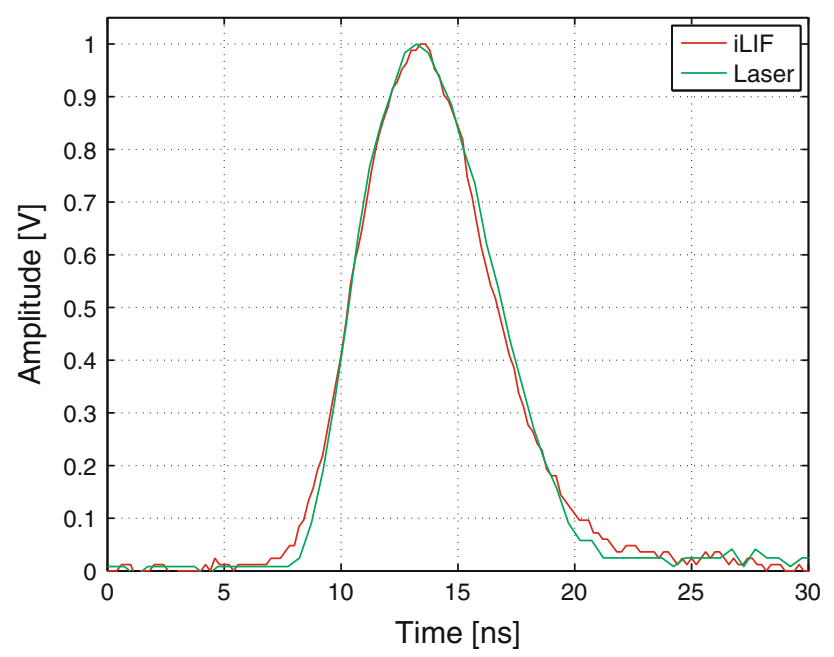

Fig. 3 The normalized average of 16 measurements of the pulse shape of both the laser and the $i L I F$ pulse

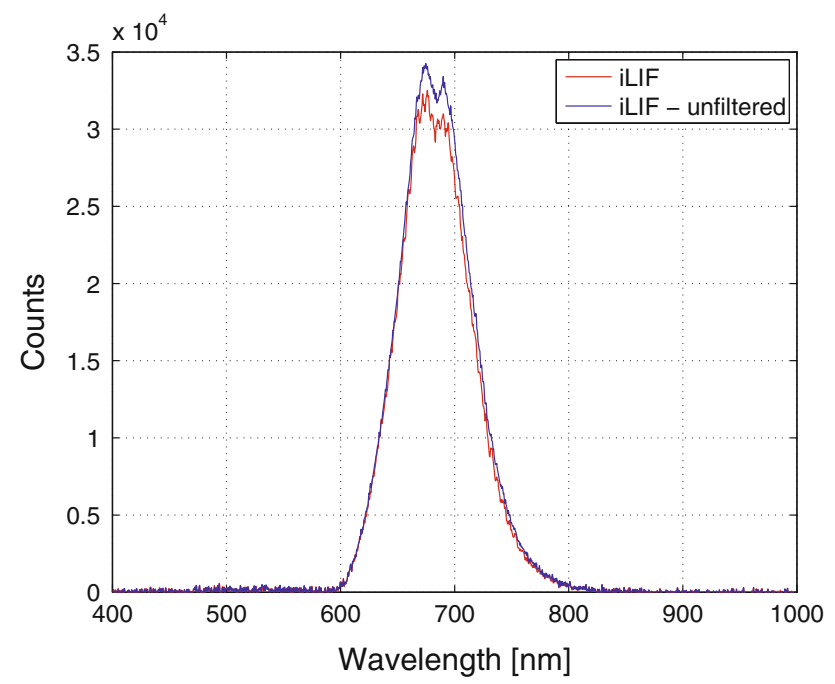

Fig. 4 Spectra of the $i L I F$ pulse with- and without the notch filter

for iLIF. The continuous xenon light source (Hella B.V.) has an aperture with a radius of approximately $10 \mathrm{~cm}$.

The test setup is schematically depicted in Fig. 5 and was designed to measure the relative intensity of all the light sources. Since the light source properties such as the beam profile, beam divergence and spectral intensity profile vary from one light source to the other, the setup was chosen to resemble a typical but simplified experimental setup for high-speed flow visualization. An aspheric lens pair collimated the light from the source onto a USAF 1951 resolution test chart (Edmund Optics). A telecentric lens (Jenoptik) was used to form a 1:1 image of a $500 \times$ $500 \mu \mathrm{m}^{2}$ square from the target onto a CCD-camera (Lumenera, LM165M, 8 bit monochrome). Neutral density
Table 1 Characteristics of the light sources

\begin{tabular}{llll}
\hline Light source & Type & $\begin{array}{l}\text { Pulse length } \\
\text { (fwhm) }\end{array}$ & $\begin{array}{l}\text { Pulse rate } \\
\text { (max) }\end{array}$ \\
\hline Laser Dye TIRlens & Pulsed & $7 \mathrm{~ns}$ & $20 \mathrm{~Hz}$ \\
Laser Dye cuvette & Pulsed & $7 \mathrm{~ns}$ & $20 \mathrm{~Hz}$ \\
HSPS Nanolite KL-L & Pulsed & $16 \mathrm{~ns}$ & $20 \mathrm{kHz}$ \\
Seoul Semicon P7 & Pulsed/CW & $1 \mu \mathrm{s}$ & $1 \mathrm{MHz}$ \\
Lumiled Luxeon Star & Pulsed/CW & $400 \mathrm{~ns}$ & $1 \mathrm{MHz}$ \\
Perkin Elmer MVS-7010 & Pulsed/CW & $200 \mu \mathrm{s}$ & $10 \mathrm{~Hz}$ \\
Olympus ILP-1 & $\mathrm{CW}$ & - & - \\
Sumita LS-M352 & $\mathrm{CW}$ & - & - \\
Schott KL1500LCD & $\mathrm{CW}$ & - & - \\
Schott Ace & $\mathrm{CW}$ & - & - \\
Hella Mega Beam Xenon & $\mathrm{CW}$ & - & - \\
\hline
\end{tabular}

a RGB 10W

b $\mathrm{G} 1 \mathrm{~W}$

filters with optical densities ranging from 2.5 to 5.0 were used to avoid overexposure of the CCD. Except for the first aspheric lens, all elements of the setup and the position of the light source were fixed in place. To optimize the light intensity and to ensure even illumination of the target, the position of the movable lens was adjusted for each light source.

For the continuous light sources, the exposure time of the camera was adjusted to get an average pixel value of the image of the square of approximately 200 out of 255 counts. This procedure was repeated for several ND-filters per light source. The recorded images were analyzed, and the results were scaled with the exposure times, the number of pulses and the optical density of the ND-filters used.

Figure 6 shows the comparison of the single pulse intensities of the pulsed light sources. Saturation behavior of the fluorescent laser dye is visible in the graph as the maximum light intensity peaks at a laser energy of $5.7 \mathrm{~mJ}$. Compared with the LED's the $i L I F$ shows a sixfold higher light intensity at a pulse length which is 2 orders of magnitude shorter. The Nanolite has a light intensity comparable with the LED's but with a pulse length similar to the $i L I F$. However, the location of the spark from the Nanolite varies per shot, causing large variations in illumination intensity and inhomogeneities between images.

The results of the continuous light sources were scaled to an illumination time of $7 \mathrm{~ns}$ to match the pulse length of the $i L I F$ and compared in Fig. 7. From the graph, the potential of the $i L I F$ becomes clear; the semi-continuous xenon flash is outperformed by two orders of magnitude and the continuous light sources are even a factor of one thousand less intense than the $i L I F$. In future research, it would be interesting to compare the $i L I F$ technique with illumination by a copper vapor laser (Oxford Lasers). Such 


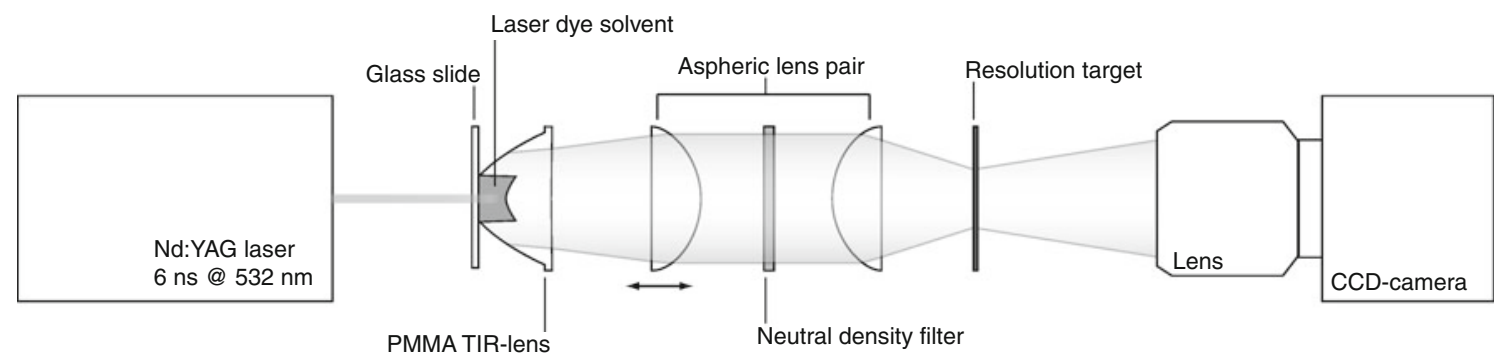

Fig. 5 Setup used for the comparison of the various light sources. The location of the first aspheric lens is adjusted to collect and collimate the light from the light source

lasers generate pulses with a relative low coherence at a very high pulse repetition frequency. However, drawbacks of these lasers are increased pulse length, lower pulse energy and higher costs.

\section{Examples}

As drop formation in inkjet printing is an almost perfectly reproducible process, single flash photography is frequently applied for its visualization (Dong et al. 2006; Hutchings et al. 2007). Here, we use this reproducible quality to demonstrate the effectiveness of the iLIF compared with 2 other flash illumination sources. These are the high-intensity Luxeon LED and the HSPS Nanolite KL-L with pulse durations of $\tau_{p}=400 \mathrm{~ns}$ and $\tau_{p}=16 \mathrm{~ns}$, respectively. The experimental setup used was equivalent to the setup shown in Fig. 2, where the inkjet print head is placed at the sample location. The incident light is focused through a collimation lens onto the region of interest. The collimation lens was chosen such that the numerical

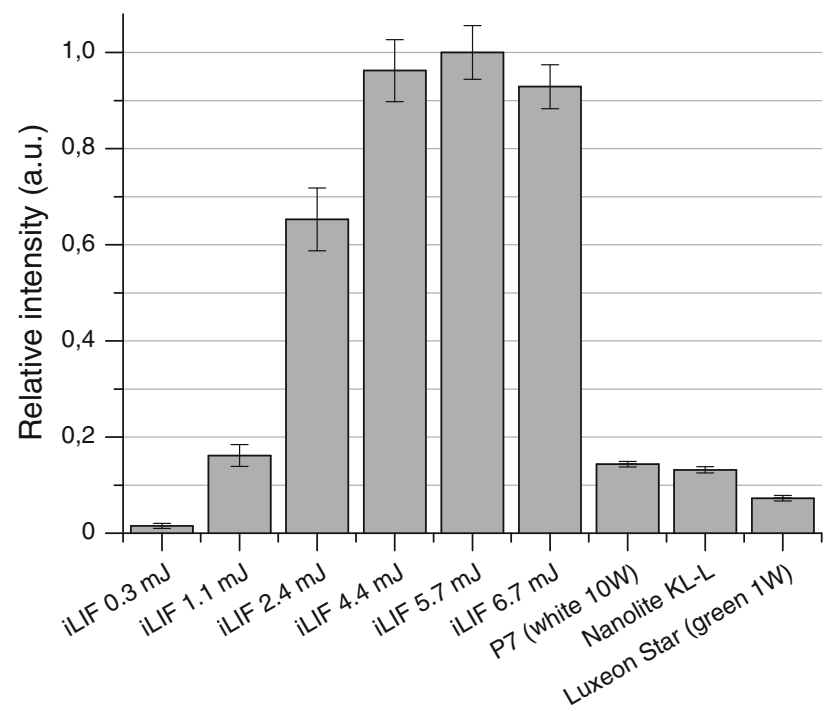

Fig. 6 Relative intensity of the pulsed light sources. The energy values indicate the laser pulse energy used to excite the laser dye aperture (NA) matches with that of the microscope objective ensuring maximal optical resolution $(r \approx 2 \mu \mathrm{m})$ and intensity (Davidson and Abramowitz 2002). The microscope (Olympus BX-30MF) is equipped with three long working distance (WD) objectives, the $L M P L F L N 10 \times$, (Olympus, WD $21 \mathrm{~mm} / \mathrm{NA}=0.25$ ), the $S M P L F L N$ $20 \times$ (Olympus, WD $25 \mathrm{~mm} / \mathrm{NA}=0.25$ ), and the SMPLFLN 50× (Olympus, WD $18 \mathrm{~mm} / \mathrm{NA}=0.35$ ). A notch filter (Semrock, NF01-532U-25) is placed before the camera to filter out any residual laser light. The camera is a Lumenera LM165 with a sensitive Sony EXview HAD CCD sensor with pixel size of $6.45 \times 6.45 \mu \mathrm{m}^{2}$, and a resolution of $1392 \times 1040$. The scale factors for the camera and the $10 \times, 20 \times$ or the $50 \times$ microscope objective combination are $640 \mathrm{~nm} /$ pixel, $320 \mathrm{~nm} /$ pixel or $129 \mathrm{~nm} /$ pixel, respectively.

The printhead is an experimental prototype developed by Océ Technologies B.V., similar to the printheads used in references de Jong et al. (2006) and Wijshoff (2010). The printhead ejects droplets with a diameter of $30 \mu \mathrm{m}$ at a typical velocity of $20 \mathrm{~m} / \mathrm{s}$ during drop formation. Some $35 \mu \mathrm{s}$ later the tip of the droplet reaches a terminal

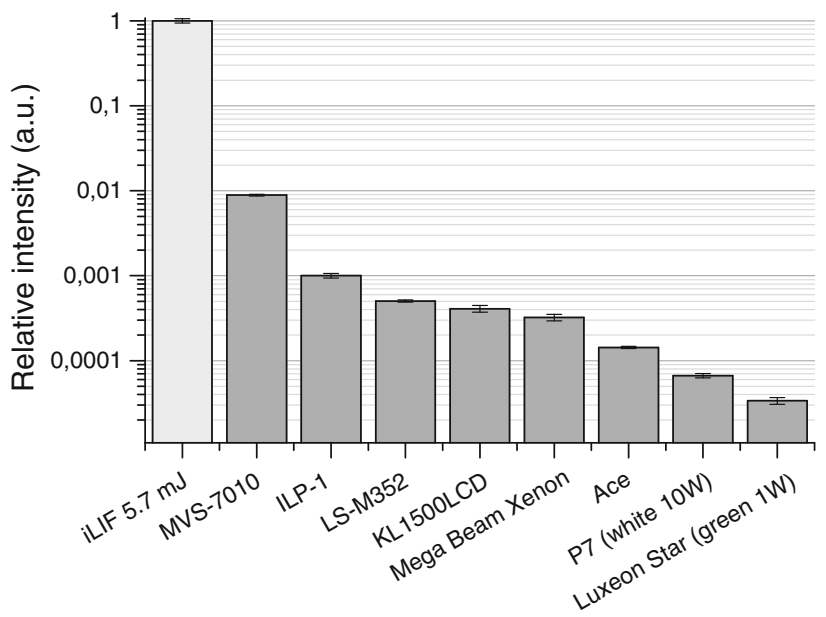

Fig. 7 Relative illumination intensities for the continuous light sources normalized to the maximum obtainable intensity of the $i L I F$ and scaled to $7 \mathrm{~ns}$ illumination time. Note the logarithmic scale 
velocity close to $6 \mathrm{~m} / \mathrm{s}$. Both stages were imaged with each of the 3 illumination sources (see Fig. 8). The left column shows the first stage of drop formation imaged with the $10 \times$ objective. The second stage where the droplet has just exited the nozzle and has slowed down to its terminal velocity is shown in the middle column, imaged with the $20 \times$ objective. The column on the right shows an enlarged view of the region where the droplet pinches off from the meniscus, imaged with the $50 \times$ objective.

The practical advantages of the combination of highintensity and short pulses of light for imaging of fast phenomena become apparent from a detailed comparison of the images in Fig. 8. First, unlike the images taken with the $i L I F$ pulse, the images in Fig. $8 \mathrm{~b}$, c required a maximal CCD gain factor of 24 for the $50 \times$ objective. As a consequence, these images have much lower contrast and a grainy appearance due to the higher noise level. This is further illustrated in the graphs of Fig. 9. Even though the images of the droplet made with the Nanolite and the iLIF appear of similar quality, Fig. 9 clearly shows that the signal-to-noise level is higher for the $i L I F$ method.

Secondly, using Eq. (4) for the indicated magnifications we find that the droplet can only be imaged without motion-blur if the illumination times are smaller than $\tau_{10}=32 \mathrm{~ns}, \tau_{20}=16 \mathrm{~ns}$ and $\tau_{50}=6 \mathrm{~ns}$. The droplet displacement during exposure $\epsilon$ is acceptable for both the $i L I F$ and the Nanolite (see Table 2); however, the pulse length of the LED is too long, resulting in motion-blur as can readily be seen in Fig. 8c.

Evidently, the characteristics of the iLIF allow both high contrast and small time resolution imaging. This is also demonstrated in Fig. 10 showing the evolution of the inkjet droplet recorded with the iLIF. The images reveal the size of a thin secondary tail between the drop and meniscus (also seen in Fig. 8c). The improved quality of the recordings, both in spatial and temporal resolution, give way to novel analysis of the drop formation, as is elaborately discussed in van der Bos (2011).
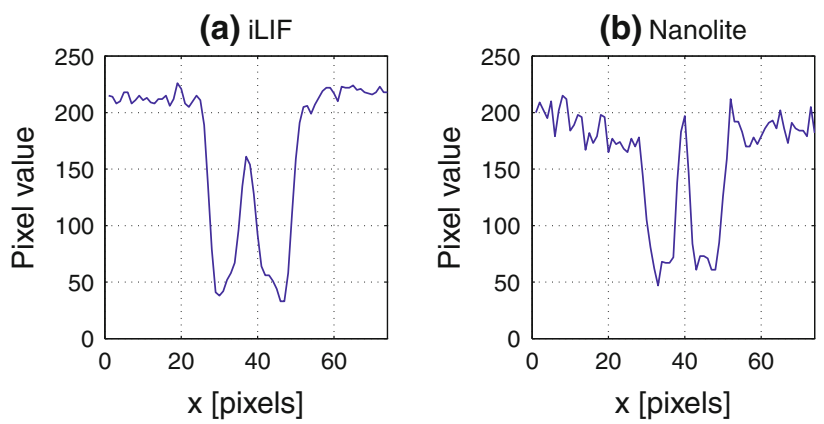

Fig. 9 Pixel values along the dashed lines in the images in Fig. 8a, b. The higher signal-to-noise ratio in the image made with $i L I F$ compared with that of the HSPS Nanolite $K L-L$ enables a more precise determination of the droplet dimensions

Table 2 Comparison of the amount of motion-blur indicated by $\epsilon$ and the relative light intensity for the imaging of a $20 \mathrm{~m} / \mathrm{s}$ inkjet droplet using 3 pulsed light sources

\begin{tabular}{llll}
\hline Light source & $M_{\text {eff }}$ & \multicolumn{1}{l}{ Rel. intensity } \\
\hline Laser dye TIR lens $\left(\tau_{p}=7 \mathrm{~ns}\right)$ & 10 & 0.22 & 1.0 \\
& 20 & 0.43 & 1.0 \\
& 50 & 1.1 & 1.0 \\
HSPS nanolite KL-L $\left(\tau_{p}=16 \mathrm{~ns}\right)$ & 10 & 0.47 & 0.16 \\
& 20 & 0.93 & 0.03 \\
Lumiled luxeon star $\left(\tau_{p}=400 \mathrm{~ns}\right)$ & 10 & 12 & 0.36 \\
& 20 & 25 & 0.10 \\
& 50 & 62 & 0.02 \\
\hline
\end{tabular}

\section{Discussion and conclusion}

The iLIF fluorescence illumination method presented here proves to be a very promising technique for flash photography of fast and small-scale phenomena. The illumination technique was evolved from an earlier applied technique were a fluorescent dye in a cuvette was used to reduce the coherence of the laser illumination. Unlike the cuvette, the geometry of the TIR-lens avoids secondary laser emission and it directs the light uniformly in the forward direction. In this way, the $i L I F$ system emits $7 \mathrm{~ns}$ light pulses with

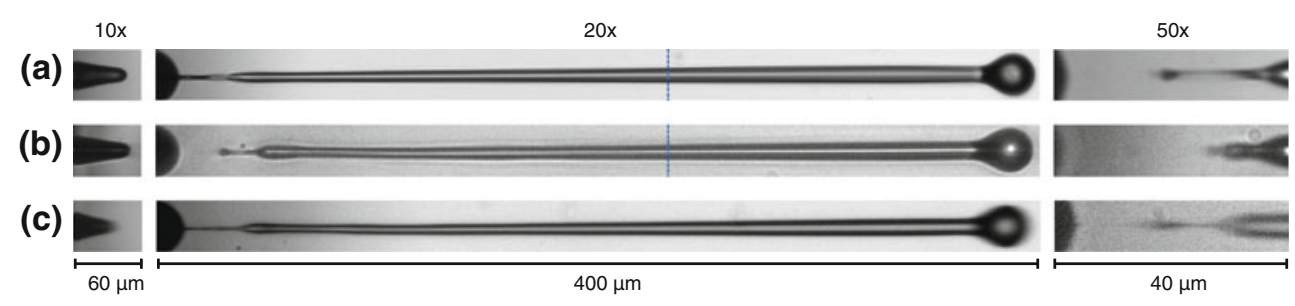

Fig. 8 Inkjet drop formation imaged using 3 different flash illumination sources and 3 different magnifications. The images illuminated with the $i L I F$ are shown in the top row (a). The second row (b) shows the recordings for which the HSPS Nanolite $K L-L$ was used and (c) shows the recording obtained with the Luxeon $L E D$. The area where the pinch-off of the droplet from the meniscus takes place is shown in the right column. The radius of the tail of the droplet is approximately $1 \mu \mathrm{m}$ in diameter. The vertical dashed lines in (a) and (b) indicate the locations for which the pixel values are plotted in Fig. 9 


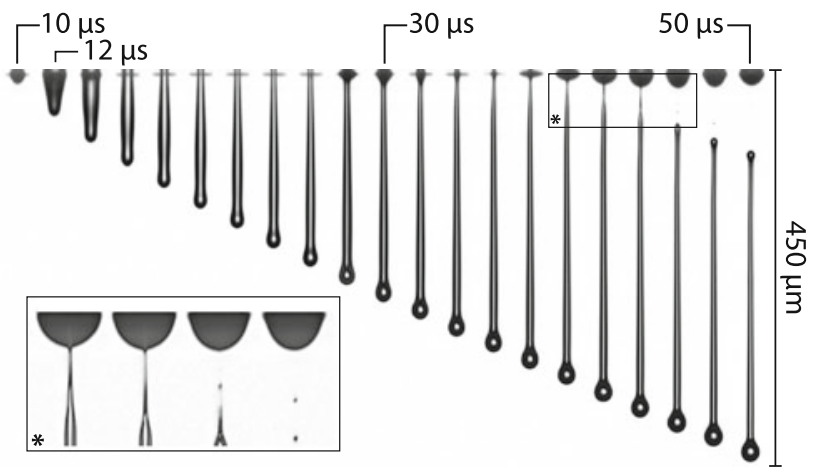

Fig. 10 Inkjet drop formation as a function of time. Here, 21 individual droplets are shown which illustrate the reproducibility of the experiment and the quality of the $i L I F$ light source. The inset shows an enlarged view of the area where the droplet pinches off from the meniscus. The background has been subtracted to create a higher contrast

low temporal coherence, thereby avoiding unfavorable effects such as speckle and diffraction. Compared with other high-intensity pulsed and continuous light sources, it shows significantly higher-intensity levels with a short pulse duration, uniform illumination and low-intensity jitter.

The characteristics of this method of illumination are determined by the properties of the laser and dye combination and the TIR-lens, which were not optimized until now. Hence, it is clear that there is plenty of space for improvements or adjustments to obtain shorter pulse lengths, higher efficiency, different wavelengths or larger apertures. The latter could be very useful for the visualization of macro-scale experiments like impact phenomena and shock-waves in fluids.

Acknowledgments We thank Rory Dijkink for his help with the laser and advice throughout the research. Additionally, we would like to thank Océ Technologies B.V. for providing the prototype inkjet print head.

Open Access This article is distributed under the terms of the Creative Commons Attribution Noncommercial License which permits any noncommercial use, distribution, and reproduction in any medium, provided the original author(s) and source are credited.

\section{References}

Chin CT, Lancée C, Borsboom J, Mastik F, Frijlink ME, de Jong N, Versluis M, Lohse D (2003) Brandaris 128: a digital 25 million frames per second camera with 128 highly sensitive frames. Rev Sci Instr 74(12):5026-5034

Davidson MW, Abramowitz M (2002) Optical microscopy. Encycl Imaging Sci Technol 2:1106-1141
Dingel B, Kawata S (1993) Speckle-free image in a laser-diode microscope by using the optical feedback effect. Opt Lett 7(18):549-551

Dong H, Carr WW, Morris JF (2006) Visualization of drop-ondemand inkjet: drop formation and deposition. Rev Sci Instr 77(8):085101

de Jong J, de Bruin G, Reinten $\mathrm{H}$, van den Berg M, Wijshoff $\mathrm{H}$, Versluis M, Lohse D (2006) Air entrapment in piezo-driven inkjet printheads. J Acoust Soc Am 120(3):1257

Edgerton HE, Killian JR (1979) Moments of vision: the stroboscopic revolution in photography. MIT Press, Cambridge

High-speed photo systeme-Nanolite, Von-Linne-Str. 12, D-22880 Wedel, Germany. http://www.hsps.com/

Hutchings IM, Martin GD, Hoath SD (2007) High speed imaging and analysis of jet and drop formation. J Imaging Sci Technol 51(5):438

Iwai T, Asakura T (1996) Speckle reduction in coherent information processing. Proc IEEE 84(5):765-781

Juhasz T, Kastis G, Suárez G, Bor Z, Bron WE (1996) Time-resolved observations of shock waves and cavitation bubbles generated by femtosecond laser pulses in corneal tissue and water. Lasers Surg Med 19(1):23-31

Lubsandorzhiev BK, Poleshuk RV, Shaibonov BAJ, Vyatchin YE (2009) Led based powerful nanosecond light sources for calibration systems of deep underwater neutrino telescopes. Nucl Instrum Methods Phys Res A 602(1):220-223

Miyashiro S, Grönig H (1985) Low jitter reliable nanosecond spark source for optical short-duration measurements. Exp Fluids 3:71-75

Nanophoton Corp., A-508, Center for Advanced Science and Innovation, Osaka University, 2-1 Yamadaoka, Suita, Osaka, Japan. http://www.nanophoton.jp/

Oxford Lasers Ltd., 8, Moorbrook Park, Didcot, Oxon, United Kingdom. http://www.oxfordlasers.com

Rau KR, Quinto-Su PA, Hellman AN, Venugopalan V (2006) Pulsed laser microbeam-induced cell lysis: time-resolved imaging and analysis of hydrodynamic effects. Biophys J 91(1):317-329

Schäfer FP, Schmidt W, Volze J (1966) Organic dye solution laser. Appl Phys Lett 9(8):306

Scholfield CN, Murdock M (1987) Pulse-modulated light source for psychometric and vision experiments. J Neurosci Methods 19(3):203-207

Shan JW, Lang DB, Dimotakis PE (2004) Scalar concentration measurements in liquid-phase flows with pulsed lasers. Exp Fluids 36:268-273

Takahashi EJ, Lan P, Mücke OD, Yasuo N, Katsumi M (2010) Infrared two-color multicycle laser field synthesis for generating an intense attosecond pulse. Phys Rev Lett 104(23):233901

Thoroddsen ST, Etoh TG, Takehara K (2008) High-speed imaging of drops and bubbles. Ann Rev Fluid Mech 40(1):257-285

van der Bos JA (2011) Air entrapment and drop formation in piezo inkjet printing. Ph.D. Thesis., University of Twente

Vasiliev RV, Lubsandorzhiev BK, Pokhil PG (2000) A nanosecond light source for scintillation-and cerenkov-detector calibration. Instrum Exp Tech (USSR) 43(4):570-572

Wijshoff $H$ (2010) The dynamics of the piezo inkjet printhead operation. Phys Rep 491(4-5):77-177

Worthington AM (1895) The splash of a drop. E. \& J.B. Young \& CO, New York

Zijlstra AG, Ohl CD (2008) On fiber optic probe hydrophone measurements in a cavitating liquid. J Acoust Soc Am 123(1):29-32 